\title{
«Cher Ami»: une lettre d'Antonin Artaud jette un nouveau regard sur ses dessins
}

\section{Giuliana Prucca}

\section{(2) OpenEdition}

1 Journals

\section{Édition électronique}

URL : http://journals.openedition.org/studifrancesi/5503

DOI : 10.4000/studifrancesi.5503

ISSN : 2421-5856

\section{Éditeur}

Rosenberg \& Sellier

\section{Édition imprimée}

Date de publication : 1 septembre 2011

Pagination : 366-378

ISSN : 0039-2944

\section{Référence électronique}

Giuliana Prucca, « «Cher Ami»: une lettre d'Antonin Artaud jette un nouveau regard sur ses dessins », Studi Francesi [En ligne], 164 (LV | II) | 2011, mis en ligne le 30 novembre 2015, consulté le 08 janvier 2021. URL : http://journals.openedition.org/studifrancesi/5503 ; DOI : https://doi.org/ERREUR PDO dans /localdata/www-bin/Core/Core/Db/Db.class.php L.34 : SQLSTATE[HY000] [2006] MySQL server has gone away

\section{(c) (†) $\odot$}

Studi Francesi è distribuita con Licenza Creative Commons Attribuzione - Non commerciale - Non opere derivate 4.0 Internazionale. 


\section{«Cher Ami»: une lettre d'Antonin Artaud jette un nouveau regard sur ses dessins}

La destinée critique de l'œuvre graphique d'Antonin Artaud pourrait voir changer son cours à partir d'une erreur de datation dans la correspondance entre les protagonistes de la scène intellectuelle et artistique parisienne de la fin des années Quarante qui gravitent autour de la figure de Jean Paulhan.

Une étude attentive et un contrôle croisé des documents à notre disposition nous ont amenés à découvrir que la lettre datée 10 janvier $1945^{1}$ au directeur de la «Nouvelle Revue Française», où Artaud dit avoir envoyé les dessins à Jean Dubuffet ainsi que celui-ci lui demande avec la lettre du 28 novembre $1945^{2}$, devrait, à notre avis, être postdatée d'un an. Ce serait le cas, par conséquent, de la lettre du peintre à l'écrivain, datée, elle aussi par erreur, 15 janvier 1945 et où Dubuffet dit ne pas avoir reçu les deux grands dessins en couleurs - mais «je voudrais bien les recevoir car cella m'intéresse grandement» - et il ajoute avoir lu Le Théâtre et son double «le mois dernier» ${ }^{3}$. Ce qui est confirmé par sa lettre à Paulhan de décembre 1945, où il utilise le verbe au présent «Je lis Le Théatre et son double» et, surtout, par la lettre du 15 janvier 1946, où il écrit toujours à Paulhan, le même jour qu'à Artaud donc et avec les mêmes mots, être grandement intéressé par les grands dessins en couleur dont parle Artaud mais je n'ai rien reçu du tout de tel», en lui retournant, par pièce jointe, la lettre de l'écrivain, qui ne peut donc remonter d'un an en arrière 4 . De plus, dans la lettre à Henri Thomas du 12 février 1946, Artaud précise qu'il a reçu «il y a quelques

(1) Lettres écrites de Rodez 1945-1946, E. C., XI, Paris, Gallimard, 1974, p. 20. La lettre est conservée dans le fonds Jean Paulhan auprès de l'IMEC (Institut Mémoires de l'Edition Contemporaine) à l'Abbaye d'Ardenne à Saint-Germain-La-BlancheHerbe près de Caen. C'est dans le cadre de notre travail de recherche qui a abouti à l'élaboration de la thèse Devenir minéral. Du mur à la poussière, soutenue à l'Università degli Studi de Turin le 11 juin 2009, que nous avons pu faire la découverte de cette fausse datation dont le présent article développe ultérieurement les conséquences. Notre travail de doctorat se veut, en effet, comme l'exploration d'un aspect du travail littéraire d'Artaud moins connu, surtout en Italie, par rapport à ses théories sur le théâtre: l'entrelacement de certains éléments fondamentaux de son écriture des années Trente et Quarante, ainsi que l'inorganique et l'informe, avec la peinture matiériste de Dubuffet et de Gutaï ou la danse Butô au Japon, les toiles et les performances de Klein et avec les installations de Kiefer, Fabre et Parmiggiani, artistes pour qui l'écrivain était souvent une précise source d'inspiration ou simplement de suggestion et pour qui la matière, et notamment celle minérale - résidu pétrifié d'un principe abstrait -, semble avoir été le matériau même pour, à la fois, la destruction du sujet de la représentation, le dépassement de la condition humaine et la construction, à sa place, d'une nouvelle «corporéité inorganique», qui voit dans l'inhumanité des choses justement une possibilité de devenir autre. En partant de la «minéralogie interne» d'Antonin Artaud et grâce à l'exemple littéraire de Joë Bousquet et, surtout, de Francis Ponge, pour qui la référence à l'écrivain marseillais a été essentielle dans l'élaboration d'une poétique capable de refaire le monde, le corps et la parole à partir d'une perspective au ras de la terre et des choses, notre but a été celui de montrer la parabole d'Artaud ainsi qu'une «incendie de silex d'os», selon les mots de Camille Bryen en son hommage, dont l'éclatement se propagerait jusqu'aux expressions artistiques les plus récentes et serait à la base donc d'un mouvement général de dépersonnalisation et d'abolition de la forme dans la matière sans nom.

(2) Correspondance, in Prospectus et tous écrits suivants IV, Paris, Gallimard, 1995, p. 101. La lettre est conservée à la Bibliothèque Nationale de France, département des Manuscrits.

(3) Ibid., p. 98.

(4) Correspondance 1944-1968, op. cit., pp. 270 et 274 . 
mois la visite d'un certain Jean Dubuffet» et que c'est seulement «après son départ» qu'il «s'est mis [...] à m'écrire de Paris, qu'il avait lu le Théâtre et son double»5. Or, la première rencontre entre Dubuffet et Artaud se passe à l'asile de Rodez en septembre 1945.

Il paraît donc invraisemblable qu'en janvier 1945 l'écrivain envoie au peintre, sans le connaître encore, ses travaux et que cela ne comporte pas une suite immédiate de leur correspondance et une familiarité plus marquée entre eux que l'adjectif «un certain» démentirait. Même si quelques lettres ont pu avoir disparu, les deux lettres de janvier 1945 seraient par contre un fait isolé, un échange fréquent, direct ou par l'intermédiaire d'autres correspondants, ne commençant qu'en automne 1945, alors que Dubuffet lui dit qu'avec Paulhan «nous nous communiquons souvent vos lettres». À partir de la fausse datation, Paule Thévenin a supposé que cette dernière phrase se référait à une série de lettres récentes qui manqueraient entre Paulhan et Artaud, du 27 janvier 1944 à celle qui date, à son avis, du 10 janvier 1945 et qui, par son ton même, ne se présente pas comme un premier message après une longue période sans échange de correspondance. Ce qui justifierait, pour elle, la présence de lettres précédentes qui pourraient être restées donc chez le peintre ${ }^{6}$. Elles ne résulteraient pourtant pas aux Archives de la Fondation Jean Dubuffet à Paris. On y retrouve par contre une lettre sans date de Paule Thévenin à un certain Hubert, qui pourrait être, très probablement, Hubert Damisch, l'éditeur des Prospectus de Dubuffet, où elle parle des lettres à celui-ci et à sa femme, publiées dans Suppôts et Suppliciations, et elle fait référence à celles qui manqueraient: «Or vous verrez qu'il y en a eu beaucoup d'autres, celle dans laquelle Antonin Artaud demandait en janvier 1945 que soit recherchée l'adresse de Catherine Chilé, celle du 13 octobre, celle où il était question des dessins de Jean Dubuffet, celle qu'en avril 1946 on faisait suivre à Lili Dubuffet, et bien d'autres qu'Artaud a écrites de Sainte Maxime et d'Ivry. C'est bien dommage qu'on ne les retrouve pas». Cela montrerait une concertation entre les deux éditeurs et expliquerait peut-être la double coïncidence de l'erreur.

Mettant en doute, d'un côté, non pas la véritable existence des lettres manquantes entre Paulhan et Artaud, mais le fait que celle du 10 janvier soit la première après l'interruption ${ }^{7}$ et réfutant, de l'autre, la thèse d'une correspondance entre Dubuffet et Artaud avant leur rencontre, notre hypothèse replacerait d'abord les deux lettres dont il est question ici dans leur trame dense de relations avec le contexte de ces auteurs entre fin 1945 et début 1946.

Outre la probabilité qu'ils se soient croisés pendant les années Vingt, tous les deux ayant fréquenté l'atelier d'André Masson rue Blomet, et à part les quelques visites à l'hôpital psychiatrique de Rodez à partir du mois de septembre 1945, Dubuffet a participé activement à la libération d'Artaud de son internement et à son retour donc à la vie. Le peintre a été présent parmi le comité d'accueil qui attendait l'écrivain à la Gare d'Austerlitz, de retour à Paris le 26 mai 1946', et il sera nommé trésorier

(5) A. Artaud, A Henri Thomas, in Lettres, in Suppôts et Suppliciations, E. C., XIV*, Paris, Gallimard, 1978, p. 77.

(6) Cf. notes in Lettres écrites de Rodez 19451946, E. C., XI, pp. 289-290.

(7) Elle est d'ailleurs envoyée chez Paulhan, rue des Arènes, comme toutes les lettres de l'année suivante, alors qu'une autre ayant la même date, c'està-dire 10 janvier 1945 , et celles immédiatement postérieures portent encore l'adresse du siège des
Lettres Françaises.

(8) Témoignage d'Ida Bourdet in Le Petit Matin d'Austerlitz / Un entretien recueilli par Pierre Chaleix, «La Tour de Feu», n. 112, décembre 1971, référé par Paule Thévenin in A. ARTAUD, Lettres écrites de Rodez 1945-1946, E. C., XI, Paris, Gallimard, 1974, pp. 356-357. Pour d'autres témoignages sur les rencontres entre Artaud et Dubuffet, voir aussi J. Prével, En compagnie d'Antonin Artaud, Paris, Flammarion, 1994. 
et gestionnaire des fonds recueillis en sa faveur et en prévision de sa subsistance. Il quittera ces «fonctions délicates et souvent contrariantes» un an plus tard, pour qu'il n'y ait plus dans leur rapport «cette question épineuse de monnaie» et afin donc de garder leur amitié désormai «acquise» ${ }^{9}$, faite de rencontres et de confrontations sur leur travail artistique, d'estime et d'appréciation réciproque qui les a amenés à exécuter chacun le portrait de l'autre et, comme on le verra, à se poser les mêmes questions sur le plan formel.

Surtout, cette nouvelle datation démonterait un apparat critique qui a été mis en œuvre par Paule Thévenin à partir de la phrase très importante d'Artaud dans sa lettre du 10 janvier à Paulhan - «Je me suis mis à faire de grands dessins en couleurs. [...] Ce sont des dessins écrits avec des phrases qui s'encartent dans les formes afin de les précipiter»- et qui a fait remonter leur naissance au début 1945, alors que, même si leur datation n'est pas sûre, ils sont attestés avec une certaine fréquence seulement dès le mois de septembre de la même année. Ce qui serait confirmé par la liste des dessins qu'Artaud même établit dans ses cahiers dès janvier 1946 et par les commentaires qui sont souvent contemporains à leur réalisation et qu'il écrit à partir de septembre 1945, probablement poussé par ses visiteurs, ainsi que Dubuffet l'invite à faire avec la lettre du 28 novembre 1945 pour qu'il explique sa démarche: «Si vous le voulez vous pourriez me les communiquer pour les faire photographier et je vous les rendrais ensuite; mais il serait très utile que vous les accompagniez de quelques commentaires: ceux que vous en aviez fait verbalement pour moi» ${ }^{10}$.

Ces dessins correspondraient également à ceux dont Artaud parle à Henri Thomas dans la lettre de février 1946, déjà citée, et qui «sont pleins de formes larvées, dans l'achoppement même du trait de crayon ${ }^{11}$. Il est significatif que, juste après cette définition, l'écrivain ajoute avoir reçu la visite de Jean Dubuffet, qui, d'ailleurs, à la même époque, déclarait, dans ses Notes pour les fins-lettrés, aimer «le peu, [...] l'embryonnaire, le mal façonné, l'imparfait» ${ }^{12}$. Même si le peintre n'avait pas reçu les dessins de l'écrivain, comme on l'a mentionné plus haut, il avait vu à Rodez ses «dessins sur un carnet» et, continue Artaud, après «avoir parlé avec moi d'art, de théâtre, de poésie et avoir, de ma bouche, tout appris sur mon internement, [...] il avait lu le Théâtre et son Double et avait été très surpris d'y retrouver toutes ses idées à lui sur l'art et le théâtre» ${ }^{13}$. L'écrivain recopie ici les mêmes mots employés par le peintre dans la lettre que celui-ci lui avait envoyée le 15 janvier 1945, selon l'édition de Damisch, ou bien 1946, selon notre hypothèse, justifiée encore une fois par une proximité de dates:

J'ai longuement lu le mois dernier votre livre «Le Théâtre et son double». Cette lecture m'a donné un considérable plaisir et je suis content de vous dire à quel point extrême j'aime et admire cet ouvrage. J'ai été surpris d'y retrouver toutes les conceptions qui sont aussi depuis longtemps les miennes propres sur l'art et sur le théâtre, aussi j’y souscris entièrement. Ce sont des idées du même ordre, très parentes de vos idées sur le théâtre, qui guident et conditionnent mes travaux de peinture. C'est vous dire à quel point votre admirable livre m'est cher et précieux ${ }^{14}$.

(9) J. Dubuffet, À Antonin Artaud [Dimanche, 4 mai 1947], lettre conservée aux Archives de la Fondation Dubuffet. À la Fondation il est possible de consulter aussi le livre des comptes tenus par Dubuffet de juin 1946 à juin 1947, avec toutes les dépenses et les recettes pour Artaud. Les versements en sa faveur proviennent souvent des dons ou des ventes de tableaux par l'intermédiaire du galeriste Pierre Loeb, à qui, d'ailleurs, le peintre confiera ensuite ses fonctions de trésorier (cf. leur échange épistolaire entre avril et mai 1947 in J. Dubuffet - J.Paulhan, Correspondance 1944-
1968, Paris, Gallimard, 2003, pp. 791-797).

(10) Prospectus IV, op. cit., p. 101.

(11) A. Artaud, A Henri Thomas [Rodez, 12 février 1946], E. C., XIV*, p. 77.

(12) J. Dubuffet, Notes pour les fins-lettrés, in Prospectus aux amateurs de tout genre, in Prospectus et tous écrits suivants I, Paris, Gallimard, 1967, p. 88 [note: L'art qui ne connaît pas son nom].

(13) A. Artaud, A Henri Thomas, E. C., XIV*, p. 77.

(14) Correspondance, in Prospectus et tous écrits suivants $I V$, op. cit., p. 98. 
Admiration qui est confirmée encore par une carte postale que Dubuffet envoie de Lausanne à Paulhan le 11 décembre 1945, où il écrit être en train de lire l'essai d'Artaud et être «émerveillé d'y trouver justement les mêmes idées que les miennes» ${ }^{15}$.

Il est peut-être révélateur de mentionner ici que le texte de Dubuffet L'auteur répond à quelques objections, mieux connu sous le titre de Rébabilitation de la boue, a aussi été publié dans le Prospectus aux amateurs de tout genre de 1946. Ce premier recueil du peintre a paru chez Gallimard, dans la collection «Métamorphoses» dirigée par Jean Paulhan, la même qui, en 1938, avait édité Le Théâtre et son double et l'avait réimprimé en 1944, version dans laquelle Dubuffet a pu sans doute le lire.

Ce qui peut l'avoir frappé dans cette lecture, c'est justement l'importance octroyée par Artaud, pour la complétude et la réussite d'un spectacle, aux aspects techniques, à «tous les moyens d'expression utilisables sur scène, comme musique, danse, plastique, pantomime, mimique, gesticulation, intonations, architecture, éclairage et décor» que tout le monde traite généralement de «partie basse» et que l'écrivain considère par contre comme «spécifiquement théâtral dans le théâtre», c'est-à-dire comme son matériau, dans le sens qu'il entend le subjectile. Afin de transformer le théâtre occidental en «poésie dans l'espace», de lui rendre son langage concret et de lui faire rejoindre ainsi l'esprit des spectacles balinais ou, par exemple, de «la vieille magie cérémonielle» ${ }^{16}$ mexicaine, il faut changer d'abord de destination à la parole, la détourner de son sens verbal pour un usage plus matériel et spatial sachant mieux parler à la sensibilité par contact direct, par contagion presque, ce qui lui ferait retrouver son rôle communicatif. À cet effet, Artaud rédige un Premier Manifeste du Théâtre de la Cruauté ${ }^{17}$, où il indique point par point toutes les astuces pour mettre en valeur la mise en scène directe plutôt que le texte écrit, le «dialogue fixé à priori» ${ }^{18}$. Il en résulte une vision très plastique et picturale du théâtre, où la matière est représentée cette fois par le geste qui «part de la NÉCESSITÉ de parole beaucoup plus que de la parole déjà formée» ${ }^{19}$ et d'un «langage physique à base de signes et non plus de mots» ${ }^{20}$. Si «dans ce théâtre toute la création vient de la scène» ${ }^{21}$ ainsi que dans la peinture elle vient du matériau pictural, ce qui se dessine carrément sur le plateau c'est une impression de mouvement esquissé, inachevé, improvisé. Cela ne pouvait pas déplaire à Dubuffet qui gribouillait des lignes «obéissant à un procédé de forces expulsées plutôt que dessiné»»"22, ainsi qu'Artaud lui avait précisément écrit, des lignes sommaires projetées sur ses tableaux «dans tous les sens du hasard, de la possibilité, de la chance, ou de la destinée» ${ }^{23}$, et qui considérait justement l'espace comme rien d'autre qu'un théâtre de gestes ${ }^{24}$.

S'en prendre au support, au subjectile dans le cas de la peinture et à la scène dans le cas du théâtre, signifierait donc conduire une démarche vers le bas, mettre en branle une procédure entropique, se servir d'instruments «maladroitement dessinés pour que l'œil qui les regarde tombe» ${ }^{25}$. C'est pour cela qu'Artaud pourrait s'inscrire

(15) J. Dubuffet - J.Paulhan, Correspondance 1944-1968, op. cit., p. 270.

(16) A. ARTAud, La Mise en scène et la métaphysique, in Le Théatre et son double, in Euvres, Paris, Gallimard/Quarto, 2004, pp. 525-526.

(17) ID., Le Théâtre de la Cruauté. Premier Manifeste, ibid., pp. 558-565. Texte paru dans «La Nouvelle Revue Française», n. 228, $1^{\text {er }}$ octobre 1932.

(18) ID., Deuxième lettre sur le langage. A Jean Paulban [Paris, 28 septembre 1932], ibid., p. 573.

(19) ibid., p. 572.

(20) ID., Sur le Théâtre Balinais, ibid., p. 536 et Le Théâtre de la Cruauté. Second Manifeste, ibid., p. 581 .

(21) ibid., p. 540

(22) Phrase retranscrite par Dubuffet dans sa lettre à l'écrivain du 28 novembre 1945, in Prospectus et tous écrits suivants IV, op. cit., p. 101.

(23) A. ARTAud, Le visage bumain [juin 1947], in Euvres, op. cit., p. 1535.

(24) M. LoreAu, Catalogue des travaux de Jean Dubuffet. Fascicule X: Vaches-Petites statues de la vie précaire, Genève, Weber Éditeur, 1969, p. 10.

(25) A. Artaud, Le tombeau de tout qui attend [mars 1946], in Euvres, op. cit., p. 1041. 
dans «la destinée de l'informe», même avant l'œuvre de Dubuffet qui, le premier, en avait pourtant formulé la définition. Dans leur essai, Rosalind Krauss et Yve-Alain $\mathrm{Bois}^{26}$ insistent, à plusieurs reprises, sur la thèse que l'informe, avant d'être le résultat d'un effacement de la figure et d'une simple abstraction, est d'abord une opération qui amène à un glissement de sens, un acte performatif qui inflige un coup bas aux processus de la formation, à la tentative d'informer la matière, d'assimiler toute chose à une forme fixe. Et ce mouvement contre la forme est d'abord une chute, une force de gravité, une précipitation.

Déjà dans ses comptes rendus de jeunesse, Artaud montre qu'il préfère, entre autres, l'«art lourd et bestial» ${ }^{27}$ de Lucas Cranach, un art donc qui opère un abaissement, qui sert, selon la définition d'informe que Bataille donne dans le dictionnaire de Documents, à «déclasser $»^{28}$, de la forme à la matière au matériau même, jusqu'à ce que toute chose n'ait que l'apparence d'une «araignée», d'un «ver de terre» ou d'un «crachat». Artaud toutefois se pousse encore plus loin dans cette précipitation. Sa parole, ou son trait, n'est pas seulement un acte performatif, mais aussi perforateur; il ne reste pas à même le papier ou la toile, il va chercher sa matière au-delà, «là où le dessin / point par point / n'est que la restitution d'un forage, / de l'avance d'une perforeuse / dans les bas-fonds du corps sempiternel latent ${ }^{29}$. Depuis les Fragments d'un Journal d'Enfer, il «sait ce que l'apparition de cette matière signifie et de quel souterrain massacre son éclosion est le prix $\rangle^{30}$. Il en fera directement l'expérience plus tard, d'abord avec ses «sorts», ou «gris-gris», espèces d'actes magiques au pouvoir prémonitoire et incantatoire ou sortes d'injures, de revendications sous forme de lettre et de message, souvent maculées, trouées et brûlées «avec une allumette après les avoir aussi méticuleusement dessinées» ${ }^{31} ;$ puis, justement avec ses dessins de Rodez et ses portraits d'Ivry, qui portent les traces épaisses «des coups de crayon comme des coups de couteau» ${ }^{32}$. Si «la page est salie et manquée, le papier froissé» ${ }^{33}$, il faut encore «descendre dans [le] charnier» ${ }^{34}$ des mots, jusqu'à «la putréfaction de la vie» ${ }^{35}$, puisque s'attaquer au subjectile, c'est également attaquer le sujet, entamer la surface du support de l'écriture ou de la peinture aussi bien que celle de la peau, le papier lacéré ou pointillé métaphorisant les blessures du corps et renvoyant ainsi aux profondeurs de l'être, à l'homme et à ses viscères: «Les gris-gris pour en revenir à l'homme» ${ }^{36}$. Et cela, ainsi qu'Artaud écrit dans un des derniers commentaires à ses dessins le 31 janvier 1948, par «des notes, des mots [...] ardents, corrosifs, incisifs, jaillis de je ne sais quel tourbillon de vitriol sous-maxillaire, sous-spatulaire, [...] qui feront leur apocalypse car ils en ont trop dit pour naître et trop dit en naissant pour ne pas renaittre et prendre corps alors authentiquement $\gg^{37}$.

(26) L'informe: mode d'emploi, Paris, Editions du Centre Georges Pompidou, 1996.

(27) A. ARTAud, Le peintre le plus représentatif du génie de la race et le sculpteur [1922-1924], in A propos de la littérature et des arts plastiques, E. C., II, Paris, Gallimard, 1980, pp. 217-218.

(28) G. Bataille, Informe, «Documents», n. 7, vol. I, année 1929, Paris, Jean-Michel Place, 1991, p. 382 .

(29) A. Artaud, Dix ans que le langage est parti [avril 1947], in Euvres, op. cit., p. 1514.

(30) E. C., I*, Paris, Gallimard, 1976 et 1984, p. 119.

(31) ID., Les figures sur la page inerte, [février 1947], in Euvres, op. cit., p. 1467.
(32) ID., L'Ombilic des Limbes, E. C., I*, p. 61.

(33) ID., Ce dessin est une tentative grave, [fin janvier 1946], in Euvres, op. cit., p. 1039. Commentaire du dessin La machine de l'être ou Dessin à regarder de traviole.

(34) ID., Cahiers du retour à Paris. Août-septembre 1946, E. C., XXIII, Paris, Gallimard, 1987, p. 311.

(35) ID., Fragments d'un Journal d'Enfer, CE. C., I*, p. 117.

(36) ID., Dix ans que le langage est parti, op. cit., p. 1515 .

(37) ID., Ce ne sont pas des dessins..., in A. DE la Beaumelle - N. Cendo, éd., Antonin Artaud. CEuvres sur papier, catalogue d'exposition, Réunion des Musées Nationaux, Marseille 1995, p. 63. 
Cette «instance d'un travail souterrain, d'une activité insensée» ${ }^{38}$ qu'est l'informe opéré par Artaud entraîne l'art vers «quelque chose entre la pourriture et le phosphore» ${ }^{39}$. «Des devenirs-moléculaires souterrains débordent les limites du système signifiant» ${ }^{40}$, telle que la «ligne qui germe» de Klee créant des «palaces microscopiques de la proliférante vie cellulaire $\gg^{41}$ dont l'organisation pourtant n'aboutit jamais à la figure, mais au «fœetus» ${ }^{42}$, à quelque chose qui s'échappe toujours à l'absorption en forme et qui se laisse juste «sous-entendre» comme toute grande poésie. Cela n'amène pas forcément à des œuvres abstraites. Deleuze voyait déjà le «naufrage de la forme» ${ }^{43}$ dans les peintures de Goya, Rembrandt, Redon et Bacon ainsi que la «catastrophe optique» et l'«effondrement des coordonnées linguistiques» ${ }^{44}$ au sein de l'œuvre d'Artaud même, qui, d'ailleurs, ne montre pas de dessins non-figuratifs.

Paule Thévenin, qui, avec Jacques Derrida, a établi la datation de ces dessins, en situerait quatre en janvier $1945^{45}$. Par ce fait, ils resteraient, eux aussi, un fait isolé dans la production de l'artiste. Il n'y aurait pas en effet d'autres dessins entre ces derniers et ceux exécutés à partir du mois de septembre. Ce qui est difficile à croire, comme la phrase d'Artaud à Paulhan «Je me suis mis à faire de grands dessins...» présupposerait, au contraire, une continuité, une volonté de poursuivre cette nouvelle expérimentation qu'il exprime, quelques lignes plus loin, en ajoutant: «et j'en ai achevé plusieurs autres». Deux de ces quatre dessins, L'être et ses foetus et Jamais réel et toujours vrai... (ill. n. 1 et n. 2), qui répondent assez bien, selon Thévenin, à la définition de «dessins écrits» et doivent donc dater de cette période, n'apparaissent dans aucune des sept listes de dessins établies par Artaud dans ses cahiers. D'après l'éditrice, le premier pourrait s'agir du dessin intitulé Les Pieds d'Iacchus, qui apparaît dans la liste du cahier 50 de janvier 1946 et qui est, comme celui-ci, destiné à Catherine Chilé. C'est le cas aussi du troisième dessin qui porte l'inscription « Catherine », non intitulé, mais qui serait celui désigné comme La (ou le) pendu(e) dans les listes des dessins de février et avril 1946. Il pourrait se confondre avec Dépendre corps, figurant dans la liste de janvier et dont la description au même titre se trouve dans le cahier 32 datant du mois de septembre 1945 et correspondrait aux éléments de La

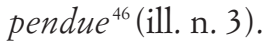

Catherine Chilé serait un mélange entre une de ces «filles du cœur» faisant partie de la famille mythique qu'Artaud s'était recréée à Rodez à partir surtout de ses ancêtres et de ses amies, et une personne réellement vécue, le Docteur Séguin, qui, d'après lui, se serait nommée Catherine, et qui aurait publié des poèmes dans «La Nouvelle Revue Française» en 1935, avant de devenir infirmière, puis médecin à l'hôpital Saint-Jacques de Paris. Par une lecture entrecroisée des lettres d'Artaud à ses différents correspondants pendant cette période, on comprend que retrouver la trace de Mlle Catherine Séguin-Chilé, c'est une question qui l'occupe, à vrai dire, vers la fin de 1945, à partir du mois de septembre et non pas avant. Cette préoccupation se reflète aussi dans ses dessins: il craint pour son sort et il se demande souvent si elle n'a

(38) H. DAmish, Fenêtre jaune cadmium ou les dessous de la peinture, Paris Seuil, 1984, p. 136.

(39) A. ARTAUD, Le peintre le plus représentatif du génie de la race et le sculpteur, CE. C., II [1980], p. 217.

(40) G. Deleuze - F. Guattari, Mille Plateaux. Capitalisme et schizophrénie, Paris, Minuit, 1980, p. 145.

(41) H. Michaux, Passages, coll. L'Imaginaire, Paris, Gallimard, 1999 , p. 116 et 115.

(42) A. Artaud, Un peintre mental, E. C., I*, p. 240 .
(43) M. Faretra, Mostri (o spettri). Klee e Pollock. con Deleuze, in A. ARTAud et al., Il sistema della crudeltà. Gli affetti, le intensità, il linguaggio dei corpi, Milano, Mimesis, 1997, p. 114.

(44) G. DelEuzE, La peinture enflamme l'écriture [1981], in Deux régimes de fous. Textes et entretiens 1975-1995, Paris, Minuit, 2003, p. 169.

(45) J. Derrida - P. Thévenin, Antonin Artaud. Dessins et Portraits, Paris, Gallimard, 1986.

(46) Cf. A. Artaud, Cabiers de Rodez. Septembrenovembre 1945, CE. C., XVIII, Paris, Gallimard, 1983, pp. 75-76. 
pas été assassinée, car elle possède «un exemplaire d'un livre de moi que personne ne connaît plus, et qui a été détruit sous l'action de la sûreté générale, de l'intelligence service et de la congrégation de l'index. Ce livre s'appelle Letura d'Eprabi [...] et qu'elle me le rapporte et cela signifiera la fin d'une ère pour ce monde et pour la vie» ${ }^{47}$. Comme il le précise à Henri Parisot à la même période, ce livre, publié en 1934 et ayant été égaré depuis, contiendrait un élément permettant de fonder un nouveau langage et de le faire enfin vivre ${ }^{48}$. Catherine Chilé aurait «quitté Paris en mai 1945 dernier et [...] est morte d'épuisement dans un champ dans sa lutte avec les envoûtements qui voulaient l'empêcher de parvenir ici. Et je ne sais pas ce qu'on a fait de son cadavre» ${ }^{49}$. Il supplie donc tous ses amis de l'aider à la retrouver et il fera lui-même, à son retour de Rodez, les démarches nécessaires, en relevant par exemple dans le Guide Rosenwald les adresses de médecins femmes avec ce patronyme ou un patronyme proche $e^{50}$.

C'est donc à ce moment-là que se situeraient les deux lettres dont il est question ici de rétablir la date exacte: en janvier 1946 et non pas 1945. En effet, Artaud commence la lettre du 10 janvier en demandant à Jean Paulhan des nouvelles de son amie, ainsi qu'il avait déjà sollicité son aide au mois d'octobre 1945, en particulier avec la lettre du $21^{51}$. A cette fin, il ajoute à la présente: «Peut-être Jean Dubuffet pourrait-il savoir son adresse». Ce qui avait été évidemment communiqué au peintre, car celui-ci débute sa lettre à Artaud cinq jours après en disant: «Je ne connais pas cette Catherine Chilé dont vous recherchez l'adresse et je ne connais personne qui la connaisse» ${ }^{52}$.

En effet, dans les mois précédents, Dubuffet a souvent servi d'intermédiaire à l'écrivain, soit que ce dernier lui confie des lettres à remettre de sa part, comme celle destinée à Elie Lascaux ${ }^{53}$, soit que le peintre se charge de la quête d'autres «filles de cœur», Anie Faure-Besnard et Cécile Schramme, à la place de Paulhan qui se trouvait dans un mauvais état de santé à cette époque-là ${ }^{54}$. Dans une lettre à ce dernier du 3 décembre 1945, conservée aux Archives de la Fondation Jean Dubuffet et publiée ensuite par Laurent Danchin dans Artaud et l'asile $2^{55}$, on lit qu'il voudrait récupérer une grosse somme d'argent «car je veux quitter la France» et il se souvient avoir depuis 1918 un compte à la Banque de France et avoir «donné un chèque de dix millions en juillet 1937 à une amie sans ressources, Anie Besnard, laquelle depuis s'est installée 45 Quai Bourbon et ne m'a plus donné signe de vie bien qu'elle me sache dans le plus grand besoin de tout, argent, aliments et le reste. J'ai demandé à Jean Dubuffet de faire une enquête et sur elle et ce compte». On remarque au passage qu'ayant dû s'occuper de la relation entre Dubuffet et Artaud, née à cette époque,

(47) ID., A Roger Blin [Rodez, 23 septembre 1945] in Lettres écrites de Rodez 1945-1946, E. C., XI, p. 121.

(48) Cf. notamment les lettres du 22 septembre et du 5 octobre 1945, in Lettres de Rodez et Lettres complémentaires à Henri Parisot, E. C., IX, Paris, Gallimard, 1971 et 1979 , p. 172 et 205.

(49) Lettre du 4 décembre 1945, E. C., IX, p. 209.

(50) Cf. en particulier Lettres, in Suppôts et Suppliciations, CE. C., XIV*: A Jean Dubuffet et Madame Jean Dubuffet [Rodez, 29 novembre 1945], pp. 5565; A Henri Parisot [Rodez, 6 décembre 1945], pp. 72-73; A Henri Thomas [Rodez, 13 et 15 mars 1946], p. 82 et 87; A Colette Thomas [Espalion, 27 mars 1946], p. 100; A Marthe Robert [Espalion, 29 mars 1946], p. 102; enfin, dans les Lettres écrites de Rodez 1945-1946, CE. C., XI, p. 265, il y a une lettre au Docteur Jean Decqueker du 8 mai 1946, où il s'interroge encore sur «ce qu'elle est devenue».

(51) ibid., pp. 147-148.

(52) Prospectus IV, op. cit., p. 98.

(53) Cf. la lettre, jamais envoyée pourtant, du 14 novembre 1945 , où il accuse, d'ailleurs, «des armées d'envoûteurs [...] disposées de tous les points de Paris et de la terre pour empêcher Catherine Chilé $[\ldots .$.$] de venir jusqu'à moi», in Lettres écrites$ de Rodez 1945-1946, E. C., XI, p. 156.

(54) Cf. la lettre de celui-ci du 27 octobre 1945, ibid., note 2, p. 320.

(55) L. Danchin, Artaud et l'asile 2, Paris, Séguier, 1996, p. 92. 
Laurent Danchin aussi, dans son recueil d'entretiens et de correspondance autour du cabinet du Dr. Ferdière, a relevé la date erronée de janvier 1945 pour les deux lettres en question.

Enfin, en revenant sur le quatrième dessin «antidaté», L'immaculée conception (ill. n. 4), sa mention figure dans presque toutes les listes des cahiers de janvier à avril $1946^{56}$. D'après Agnès de la Beaumelle ${ }^{57}$, qui a suivi la leçon de Thévenin, cela signifierait que les listes et les commentaires auraient dû apparaître «bien ultérieurement à l'exécution de certains dessins», légitimant ainsi la date de la lettre à Paulhan, le 10 janvier 1945. Par contre, nous suggérons que ces mêmes listes et commentaires pourraient fournir, par leur proximité temporelle aux dessins, un outil pour établir une datation plus précise.

Retenir les dates 10 et 15 janvier 1946 pour les deux lettres qui nous intéressent ici ne nous permet pas seulement de mieux situer les relations entre leurs auteurs, mais cela nous fournit aussi l'occasion d'une réflexion sur l'évolution du travail d'Artaud. En effet, de cette datation que nous proposons il peut s'ensuivre une nouvelle lecture critique de l'œuvre graphique d'Artaud qui se distingue nettement de la thèse développée par Paule Thévenin dans son essai La Recherche d'un monde perdu de 1984. Toujours en supposant que la lettre date du début 1945, elle écrit: «Il faut remarquer cette notion très particulière de dessins écrits qui identifie dessin et écriture, empêche d'imaginer l'un sans l'autre, les rend indissociables, et aussi relever, la lettre de 10 janvier en donne la preuve formelle, que l'exécution des premiers grands dessins a précédé la rédaction obstinée, quotidienne, des cahiers (le premier date de février 1945) comme s'il avait fallu d'abord passer par cette forme d'écriture dessinée, d'écriture idéographique, pour que la main se réhabituât à cette fonction majeure, qui avait été la sienne bien avant l'internement, de transcrire une pensée sur du papier ou tout autre support» ${ }^{58}$.

Tout en étant d'accord avec elle sur l'imbrication inséparable, chez Artaud, de la lettre et de l'image, on ne peut plus se rallier à l'hypothèse que les dessins aient précédé la composition des cahiers. Ceux-ci se présentent plutôt comme la normale continuation de ses sorts de 1937, où petit à petit des signes graphiques tracés au crayon ou même avec du feu, venaient se dessiner entre les mots adressés à ses amis et ennemis. Les grands dessins seraient donc le développement de tout cela, comme si Artaud, après s'être exercé quelques mois à cette forme d'écriture accompagnée de petits dessins, confirmant d'ailleurs son être d'abord un écrivain, avait recouvert une certaine confiance pour la transposer sur un plus grand format, ce qui lui permettra en quelque sorte d'inverser les facteurs et privilégier, par la suite, le dessin, sans pourtant abandonner complètement l'écriture. Le rôle de plus en plus important accordé au trait graphique semble accompagner l'affirmation d'une certaine identité retrouvée. Si les premières feuilles s'offrent véritablement comme des pages écrites, chargées de signes confus, petit à petit les formes, même larvaires ou «larvées», commencent à se détacher et à se distinguer du fond, jusqu'à ces portraits de ses amis qui semblent flotter dans l'espace vide et immense de la feuille et qui seront son sujet préféré depuis son retour à Paris. Il y aurait chez Artaud une sorte de réappropria-

(56) Pour les listes complètes cf. Cabiers de Rodez. Décembre 1945-janvier 1946, E. C., XIX, Gallimard, Paris 1984, p. 223; Cabiers de Rodez. Février-mars 1946, E. C., XX, Paris, Gallimard, 1984, pp. 59-60, p. 188, pp. 199-200, p. 301; Cabiers de Rodez. Avril-25 mai 1946, E. C., XXI, Paris, Galli- mard, 1985, p. 190 et 207.

(57) Antonin Artaud. Euvres sur papier, op. cit., pp. 101-111.

(58) J. Derrida-P. Thévenin, Antonin Artaud. Dessins et Portraits, op. cit., p. 27. 
tion de l'esprit passant par le dessin de ce qui est le siège de la lucidité reconquise et restée intacte sur un corps torturé, le visage. Il est peut-être significatif de remarquer que c'est à partir de la fin du mois d'avril 1946, c'est-à-dire peu avant son départ de Rodez, qu'Artaud commence aussi à signer ses dessins et à les dater lui-même.

De ce nouveau point de vue, l'évolution de son œuvre graphique tout comme celle de son œuvre littéraire illustrerait mieux donc la parabole qui, de l'étalage d'un corps en morceaux, fragmenté par la souffrance, s'achève sur la refondation d'une nouvelle anatomie qui puisse prendre possession de la totalité du soi - corps et esprit.

J'ai connu l'être en lambeau de leurs âmes dans chaque petit os de poussière qui gagnait les ténèbres premières et de chaque petit os de poussière j'ai eu l'idée dans la musique sanglotante de l'âme de rassembler un nouveau corps humain.

Ce dessin représente l'effort que je tente en ce moment pour refaire corps avec l'os des musiques de l'âme'.

GIULIANA PRUCCA

Ces dessins font partie de ceux datés janvier 1945 par Paule Thévenin et Jacques Derrida. Datation reprise, pour La Pendue, par le Centre Pompidou qui a acquis le dessin en 2009. Selon la nouvelle datation des lettres, ils devraient aussi être postdatés d'un an. Mais leur date reste pour l'instant imprécisée entre janvier et avril 1946, moment où ils font leur apparition dans les listes de dessins établies par Antonin Artaud dans ses Cabiers de Rodez. 


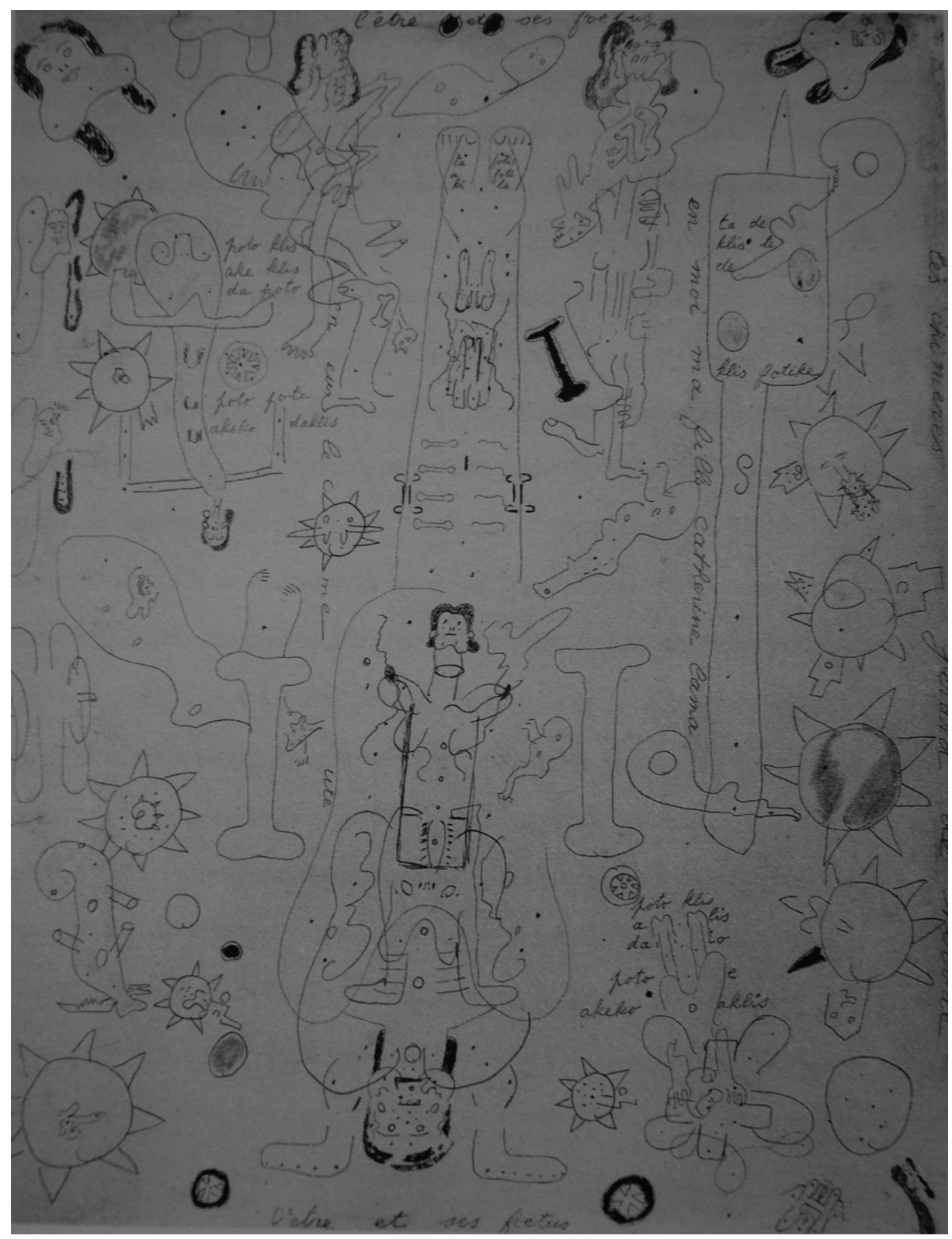

\section{L'ÊTRE ET SES FETTUS}

crayon et craies de couleur sur papier

$64 \times 50 \mathrm{~cm}$

non signé, non daté

Inscr.: «L'être et ses foetus / utérines viscères ce crime anal des êtres / les Chimères Gérard de Nerval / poto klis / ake klis / da poto / poto pote / akeko daklis / en eux le c me ute / ta totis / a foti / ki la / ta de / kis te / de / klis fotike / en moi ma fille Catherine lama »

Ancienne collection Marcel Bisiaux

Collection particulière 


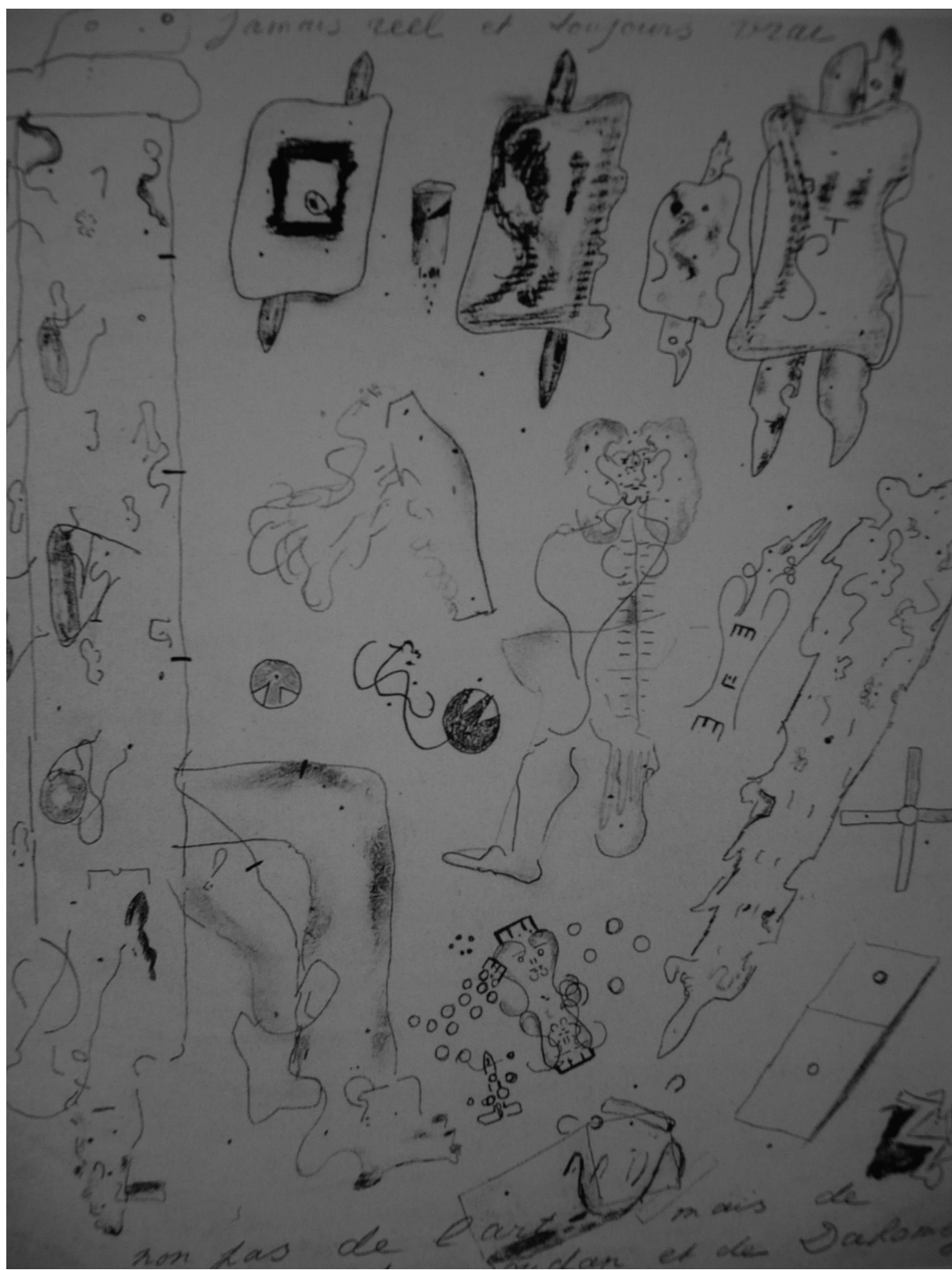

2. JAMAIS RÉEL ET TOUJOURS VRAI

crayon et craies de couleur sur papier

$64 \times 48 \mathrm{~cm}$

non signé, non daté

Inscr.: «Jamais réel et toujours vrai / non pas de l'art mais de / la ra-tée de Soudan et de Dabomey » Ancienne collection Marcel Bisiaux

Collection particulière 


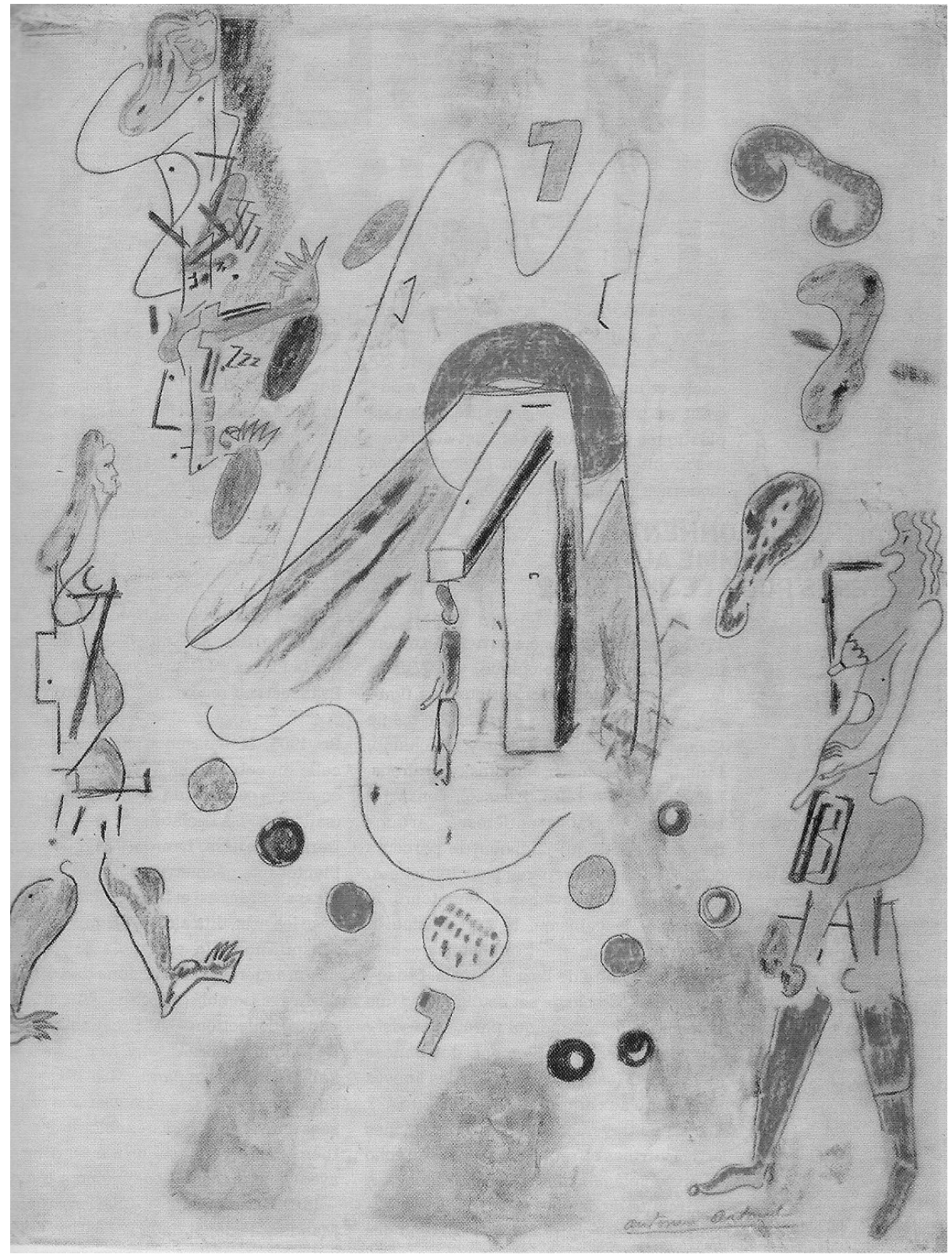

3. LA PENDUE

crayon et craies de couleur sur papier

$65 \times 50 \mathrm{~cm}$

signé en bas à droite, non daté

Inscr:: « Catherine »

Musée nationale d'art moderne - Centre de création industrielle

Centre Georges Pompidou, Paris 


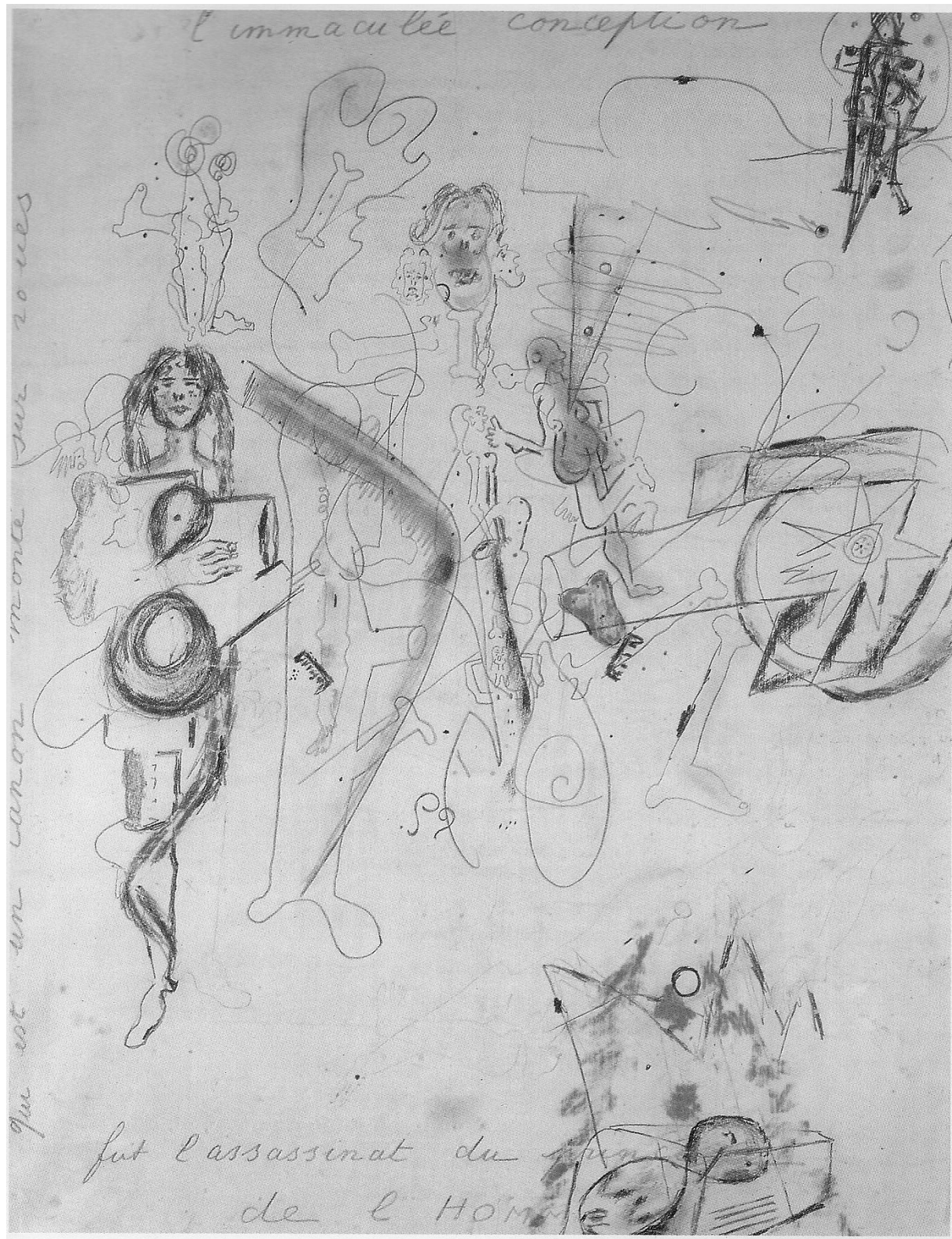

4. L'IMMACULÉE CONCEPTION crayon et craies de couleur sur papier $61 \times 48 \mathrm{~cm}$

non signé, non daté

Inscr:: "L'Immaculée conception / fut l'assassinat du principe / de l'HOMME / qui est un canon monté sur roues»

Collection particulière 